\title{
Improved Trade Framework for Business
}

\author{
Ms.Pearl Maria Mascarenhas and Dr. Pushpalatha K \\ PG Scholar $^{1}$ Associate Professor ${ }^{2}$ \\ Department of Computer Science \& Engineering \\ Sahyadri College of Engineering \& Management \\ Mangaluru, Karnataka \\ India
}

\begin{abstract}
In the information modern era because of the high usage of internet on social media and mobile phones, the enormous amounts of data have become available on hand to decision makers. Big data refers to datasets that are not only big, but also high in variety and velocity, which makes them difficult to handle using traditional tools and techniques. The huge growth of the mobile marketing and advertizing plays very important role because of the rapid improvements of mobile technologies \& huge growth in mobile device adoption. This created the new opportunities in mobile marketing and adverting which include real-time customer engagement, improve customer experience, send notification about the product, Build Brand Royalty, increase revenues, and drive customer satisfaction.

The challenges for the marketers and advertisers include how to analyze the large amount of data that mobile devices emit and how to derive customer engagement insights from the mobile data. The project "Improved Trade Framework for Business" addresses the challenge by developing mobile marketing analytics and advertising recommendation framework on Big data. This framework supports the advertising operations in which the data analytics techniques are used to provide advertisement of the vendor products based on collecting Data of users' interest trough mobile user profiles, access behaviors, and the users geographical location.

The project "Improved Trade Framework for Business" presents prototyping solution design as well as its application and certain experimental results.
\end{abstract}

Keywords: Map Navigation, Stakeholders, System Architecture.

\section{INTRODUCTION}

Mobile advertising has lot of opportunities as well as the challenges to advisors and marketers. Mainly unknown adoption in mobile technology, customer to stakeholders challenges due to huge consumable mobile datasets and the probable impact of mobility on digital marketing. These demands are related with the variety of the data, it may be structured or unstructured data, the uncommonness of the data and the confidentiality \& the context information associated with the data. In the mining of promoting perceptive from the cell phone user data, unless the data is thoroughly mined, there is a lot of breach between the advertisers, marketers and mobile users' notion. Data analytics are helping the advertisers and the database marketers to know thoroughly for framing the end user action and start understand the user's expectation of how end users like to communicate with them.

The project "Improved Trade Framework for Business" shows the strong demand of mobile advertising and marketing platform based on a big data analytics approach. The major contribution of the project is to propose the innovational solution based on big data analytics service framework with the support of mobile advertising. Here we focus on location-based mobile advertising system using analyzing the big data through mobile user registration, mobile application usage patterns, location-based mobile user access patterns, as well as vendor related data.

\section{SYSTEM ANALYSIS}

Although there are many existing systems for which are used for database marketing, there are many limitations for those applications depending on the algorithm they have used. In this "Improved Trade framework for Business" application to support 


\section{Pearl Maria Mascarenhas et al., Improved Trade Framework for Business}

system extensibility, we are using universal solution for location based advertisement recommendations. This system influences the recent available open source technology to handle the big data processing platform. The core processing is to extracting the user interests from the file to database which includes collaborative filtering, clustering \& classification of the data.

Proposed approach comprises of below Points:

- Processing the user interest File (JSON file \& CSV file)

- Adding \& providing Vendor information around the user location

- Adding \& providing the offers details on the products to which the user has interest.

Advantages:

1. Increases the Sales of vendors by catching the people's interest.

2. Mobile phone is personal and Handy. So at anytime the user can check the vendor details and may show the interest on the product.

3. This application is flexible for any user and on any mobile.

4. This application reduced Target errors and improved data management.

5. Improved Time relevance.

6. It provides the location awareness by providing the Vendor Location directly from the mobile app through Google maps.

\subsection{Assumptions Made in the Improved Trade Framework for Business:}

Since the API for the social media is not available, in this project "Improved Trade Framework for Business" assuming that we will receive the data of the user in the form of JSON file and CSV File. The admin of the application is responsible for importing this file and processing it in such a way that it will store in our database. Once it is saved we can fetch and process this data based on our requirement.

\subsection{Functional Requirements}

A functional requirement explains the functioning of the system or application. That is the functional requirement specifies the behavior or function of the application.

The functional Requirements of the Improved Trade frame work for Business applications are:

- $\quad$ User registration \&Login

- $\quad$ Admin \& Vendor Login

- $\quad$ Adding vendor \& offers

- $\quad$ Processing JSON \& CSV File.

- $\quad$ Displaying the Vendor details to user

- $\quad$ Sending Notification to the user

\section{SYSTEM ARCHITECTURE}

A system architecture is the visionary model with formal description of representation of a system, which defines the structure and behavior of a system.

The system architecture of the Improved Trade Framework for Business is as shown in figure 4.1.

\section{IMPLEMENTATION}

The implementation of this project involves 3 parts: user, admin and vendor depending on the operations.

\subsection{User operation}

The user needs to install the mobile application on his mobile and needs to register with the user name and password. Once registered, user will be able to login using registered user name and password.

\section{User Login}

After registration, the user will be able to login at any time to the mobile with the registered user name and password. On successful Login the user can see vendor location around user's area. 


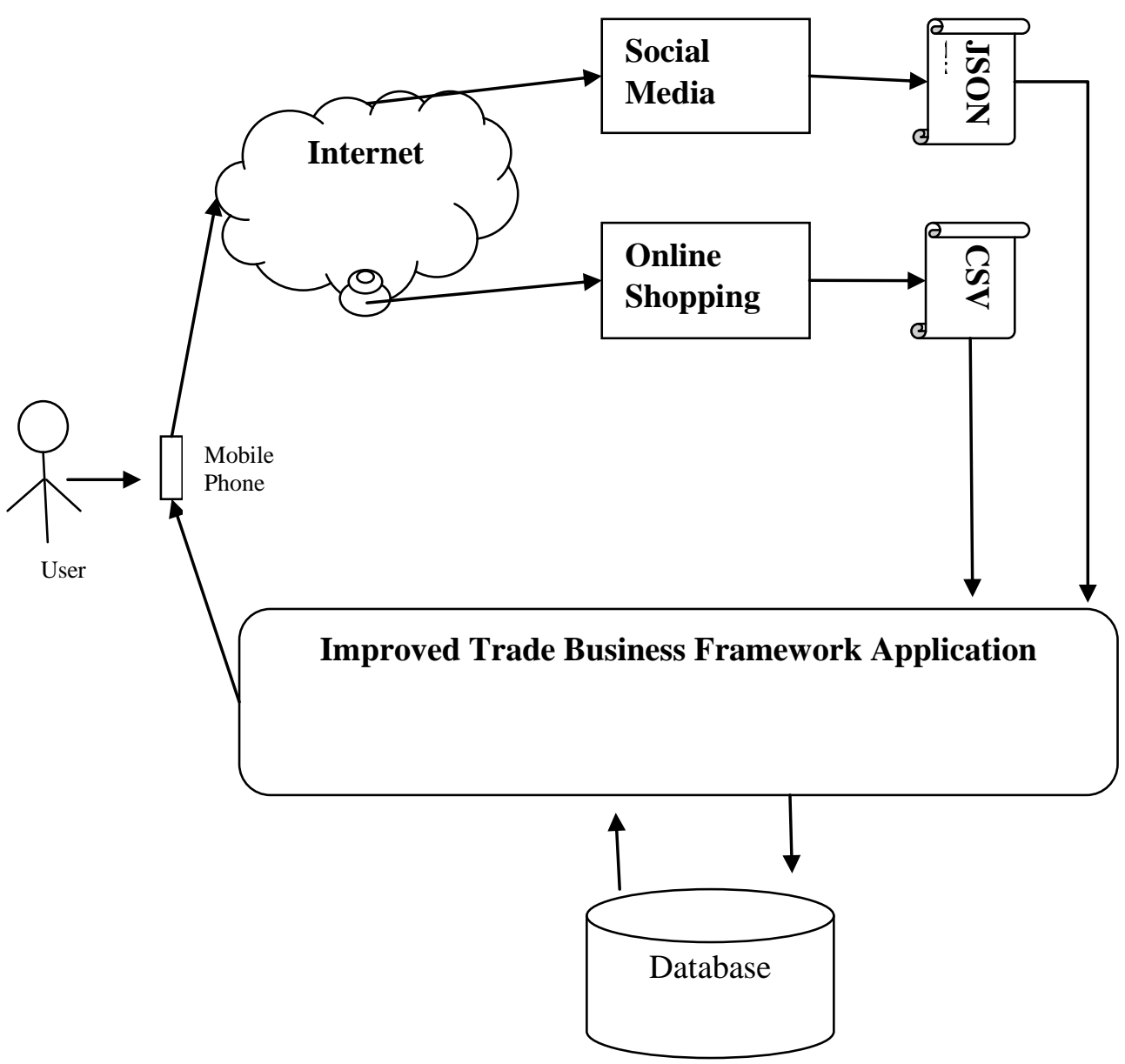

Figure 4.1: System Architecture of Improved Trade Business Framework Application.

\section{Map Navigation}

The user can view the registered vendors' location on which he has interest on his mobile application within the $10 \mathrm{~km}, 20 \mathrm{~km}$ on his phone and if the user wants to enter the radius manually, then there is a provision for entering the radius in KM. If the clicks on the vendor location, user will be able to see the offers details available in the particular vendor.

\section{Incoming Notification}

If any offers are added on that current date, the notification will be received in the mobile app. This is only if the user has interest on that particular product of the vendor.

\subsection{Admin Operation}

The admin is responsible for adding the vendor into the application. Once the vendor is added, admin is also can add the offer behalf of the vendor. Here admin also have another responsibility to extract the user interest data and to process it and sa ve it in the database, which will be used to map the user interest with the vendor product details.

\section{Admin Login}

Here we are considering the single admin, so he will know the user name \& password. Using these credentials the admin will login to the web application to perform his operations.

\section{JSON File Extraction}

Users' social network data where the user's likes/shares and search history will be obtained in JSON file format. The admin can import this file and extract the details and save it to the database.

\section{CSV File Extraction}

Users shopping history will be obtained in Comma Separated File, which the admin have the option to import it and save it to the database.

\section{Adding Vendor}

Admin has the main responsibility is to add the vendor with its details like contact number, location, the key word and item. 
Pearl Maria Mascarenhas et al., Improved Trade Framework for Business

\section{Adding Offers}

After adding the vendor, for the same vendor admin can also add the offer. Adding offer can be done from the vendor side actually. But here we have provided the option for admin also.

\subsection{Vendor Operation}

Once the vendor is added by the admin, vendor can login to the application and add the offers to his account. These offers will be displayed to the users and notification will be sent to the user if that user has the interest on this item.

\section{Vendor Login}

While adding vendor to the application the admin will provide the vendor contact number and password. Using this vendor needs to be login to the application.

\section{Adding /Deleting offers}

Once the Vendor Login to the system, vendor can add the new offers to the system and also he can delete the existing offers.

\section{CONCLUSION}

The project "Improve Trade Framework for Business" presented a recent innovative approach to supporting a location based advertisement marketing system using the current location of the user. The intention of the project is to perform the entire process of complete system testing and measuring the scalable big data advertisement processing platform in the current huge rapidly growing market. The project also provides a pilot big data analytics techniques for the vendors to view their end-users interest and the users to view the vendor's offers and location details on their mobile phones. Once this approach is examined based on the comments of the vendors and users respectively, we would improve the data analytics method of the component of our system. The scope of the current project "Improve Trade Framework for Business" is very huge in this smart phone era. In future a lot of additional features can be added to the current system to achieve high performance and scalability along with the support of realtime processing. In the future, we plan to provide online and offline modeling and training of datasets to enrich mobile user experiences.

\section{REFERENCES}

[1] Google, “Android, the world's most popular mobile platform”, [Online], 11 Jan 2015.

[2] Chuck Jones, “Why 10 Million iPhones Means A Lot More Than 9 Million”, Sep 2013.

[3] James Manyika, et al., "Disruptive technologies:Advances that willtransform life, business, and the global economy", May 2013.

[4] Cohen H. "How Your Audience Uses Mobile Now.".

[5] Martin Kihn and Mike McGuire, “Gartner Webinars, Mobile Marketing and Data-Driven Marketing”, Research VP,14 May 2014.

[6] Giamas Alex, “Spark, Storm and Real Time Analytics.”, 2014.

[7] Jones M. T. "Spark, an alternative for fast data analytics”, 2012.

[8] Kevin Normandeau "Beyond Volume, Variety and Velocity is the Issue of Big Data Veracity", 2013. 